\title{
Biodegradability and transformation of human pharmaceutical active ingredients in environmentally relevant test systems
}

\author{
Silvia Berkner • Claudia Thierbach
}

Received: 11 March 2013 / Accepted: 23 May 2013 /Published online: 14 June 2013

(C) The Author(s) 2013. This article is published with open access at Springerlink.com

\begin{abstract}
Human pharmaceutical active ingredients that are orally or parenterally administered may be metabolised in the body and after excretion may be further transformed in the receiving environmental compartments. The optimal outcome from an environmental point of view-complete mineralisation -is rarely observed. Small molecule pharmaceuticals are commonly not readily biodegradable according to Organisation for Economic Cooperation and Development (OECD) 301 tests. However, primary transformation is often observed. To gain information on the transformation of active ingredients in the environment, long-term studies like transformation in aquatic water/sediment systems according to OECD guideline 308 are required for the environmental risk assessment for human active pharmaceutical ingredients. Studies received until mid 2010 as part of the dossiers for marketing authorisation applications were evaluated concerning transformation products. The evaluation revealed that in $70 \%$ of the studies, at least one transformation product (TP) is formed above $10 \%$ of the originally applied dose, but in only $26 \%$ of the studies are all TP identified. The evaluation also revealed that some TP of pharmaceutical active ingredients show a considerably longer $\mathrm{DT}_{50}$ compared to the parent compound. An example is the TP (val)sartan acid that is formed from an antihypertensive compound.
\end{abstract}

Keywords Transformation · Pharmaceuticals .

Biodegradation - Activated sludge $\cdot$ Water/sediment system . OECD guidelines

Responsible editor: Hongwen Sun

S. Berkner $(\bowtie) \cdot C$. Thierbach

Unit IV 2.2 Pharmaceuticals, Washing and Cleansing

Agents and Nanomaterials, Federal Environment

Agency, 06844 Dessau, Germany

e-mail: silvia.berkner@uba.de

\section{Introduction}

Human pharmaceutical active ingredients (AI) are being consumed in increasing amounts worldwide. The high amounts consumed combined with the desired stability and resulting persistency of the compounds have led to their ubiquitous presence in water bodies (Hughes et al. 2013). This has triggered concerns about the ecotoxicological effects and the potential for the contamination of groundwater and raw drinking water. Therefore, since 2001, an assessment of environmental fate and effects is a mandatory part of the procedure for the marketing authorisation for human pharmaceuticals (EC 2001). The assessment is based on estimating the exposure of the environment and on experimental studies to characterise the fate and effects of pharmaceutical AI in the environment (EMA 2006).

The most common pathway into the environment for human pharmaceutical AI after usage by patients is via excretion, subsequent transport to a wastewater treatment plant (WWTP) and entry into the environment via the WWTP effluent or via spreading of sewage sludge on agricultural areas or its use in other open applications. Additionally, AI from topical formulations (e.g. gels, crèmes, etc.) and $\mathrm{AI}$ emitted from production and formulation facilities also contribute to the pharmaceutical compounds introduced into the environment (Fick et al. 2009; Phillips et al. 2010; Sanchez et al. 2011). During their use and their subsequent entry into the aquatic or terrestrial environment, AI are subject to metabolism and transformation. In the patient, the parent compound may be metabolised into phase I or phase II metabolites (ME). For many AI, a high fraction of the administered dose is excreted as mineralisation is rarely observed and accumulation of $\mathrm{AI}$ or its ME in the patient is (in most cases) not desired. During sewage treatment, further transformation may take place. The resulting mixture of the parent compound, ME and transformation products (TP) reaches 
different environmental compartments (e.g. rivers, lakes, sediment, soil) with either the sewage plant effluent or the spreading of sewage sludge onto land, where further transformation occurs including biotransformation and abiotic transformations like, e.g. phototransformation.

Depending on their sorption and biodegradation properties and the amount that is consumed, many pharmaceutical AI are not or only partly removed by conventional wastewater treatment technologies (Joss et al. 2006). Pharmaceuticals are just now starting to be incorporated into routine monitoring programmes for surface waters (EC 2012), but despite this lack of consistent monitoring efforts, it becomes clear from many studies that pharmaceutical AI and their ME and TP are widely detected in surface water and in some cases also in groundwater or raw water for drinking water production (Ternes 1998; Heberer 2002; Kolpin et al. 2002; Bergmann et al. 2011; Hughes et al. 2013).

$\mathrm{ME}$ and transformation products are covered in the environmental risk assessment by the so-called total residue approach (EMA 2006). This approach assumes that the parent compound, ME and TP show the same behaviour and effects in the environment. In many cases, this approach might be valid, but in some cases, the ME or TP actually might be of higher concern than the parent compound, and therefore, further information is required to comprehensively conduct an environmental risk assessment. ME or TP might be of higher (eco-)toxicological concern than the parent. Strategies to deal with this concern have been described (Escher et al. 2006; Escher and Fenner 2011). However, if TP are of concern because they are more persistent and/or more mobile than the parent compounds cannot be covered by the proposed testing scheme. Information on the persistence of a TP should be available from simulation type studies conducted as part of the environmental risk assessment, e.g. studies on transformation in water/sediment systems according to Organisation for Economic Cooperation and Development (OECD) guideline 308. Additionally, information on the mobility of the TP, e.g. their adsorption/desorption behaviour in relevant matrices, is required to estimate their risk for groundwater or raw drinking water contamination. It is important to know the identity of the respective ME or TP to be able to include it into monitoring programmes. Currently, for human pharmaceutical AI that are not readily biodegradable according to tests following OECD guideline 301, more detailed studies on transformation in water/sediment systems according to OECD guideline 308 have to be conducted (EMA 2006; OECD 2002). The guideline requires screening for $\mathrm{TP}$, to quantify their amount, to determine the $\mathrm{DT}_{50}$ (time required until $50 \%$ of the substance has dissipated) and to identify the TP that are formed at more than $10 \%$ of the applied dose.

The aim of this study was to evaluate information from the environmental risk assessment for human pharmaceutical AI concerning biodegradability and transformation in water/sediment systems. Studies conducted according to OECD guideline 301 on ready biodegradability (OECD 1992) and OECD 308 on transformation in water/sediment systems (OECD 2002) were analysed.

First, an overview was gained on how often TP are observed in these studies. Furthermore, the quality of studies concerning detection, quantification, identification and determination of $\mathrm{DT}_{50}$ for $\mathrm{TP}$ was evaluated. Additionally, it was studied whether TP are just transient phenomena or if there are indications of a longer persistence in the environment compared to the parent compounds. Emphasis was put on identifying specific problems that complicate the interpretation of results from OECD 308 studies from pharmaceutical AI. A comparison of results from OECD 301 tests on determining ready biodegradability and results from OECD 308 water/sediment systems was also conducted.

Based on the different evaluations, recommendations for conducting transformation studies for the aquatic environment are derived together with conclusions for the assessment of the fate and effects of TP in the environmental risk assessment of pharmaceutical AI.

\section{Materials and methods}

The evaluation is based on all available OECD 301 and OECD 308 type studies that have been submitted up to mid 2010 as part of the dossiers for marketing authorization applications as required according to EMA (2006). The OECD 301 guideline describes several methods to determine ready biodegradability of substances. For human pharmaceutical AI, the OECD 301 B test is recommended (EMA 2006) as it delivers unambiguous results on mineralisation and circumvents problems which might arise due to sorption of analytes to test vessels. In the test, diluted activated sludge from a wastewater treatment plant is incubated with (unlabelled) test substance, and the $\mathrm{CO}_{2}$ evolution is measured and compared to the theoretical maximal $\mathrm{CO}_{2}$ evolution expected for the test compound, corrected for the $\mathrm{CO}_{2}$ evolution observed in blank samples. A substance is classified as readily biodegradable if after 28 days more than $60 \%$ of the theoretically observable $\mathrm{CO}_{2}$ has formed.

The OECD 308 guideline describes a simulation test used to assess the transformation of chemicals in the environment. Details can be found in OECD (2002). Briefly, the test involves incubation of a (radio-labelled) test substance, e.g. a pharmaceutical AI, in a test vessel filled with sediment and water at a ratio of 1:3 to $1: 4(\mathrm{v} / \mathrm{v})$ sampled from the same location from either a river or a lake. The test setup according to the guideline consists of four different systems, two aerobic and two anaerobic systems. For pharmaceuticals, information on anaerobic systems is usually not received (EMA 2010); therefore, only aerobic studies were evaluated. 
The test sediments have to fulfil specific criteria concerning their organic carbon content and texture, and the test system has to be characterised concerning $\mathrm{pH}$, particle size distribution, TOC, microbial biomass and redox potential. The test substance is added to the water phase of the system, and incubation is started. Parameters measured are the partitioning of the test substance (and TP) between water and sediment, mineralisation of the test substance (only if radio labelled), non-extractable residues in the sediment (only if radio labelled), the amount of test substance and TP at different sampling time points. A screening for the formation of TP has to be included, and detected TP have to be quantified. TP present at or above $10 \%$ of the initially applied dose or those that do not show a clearly decreasing behaviour in the end of the study have to be identified. Additionally, by fitting an appropriate kinetic model to the data points, $\mathrm{DT}_{x}$ (usually $\mathrm{DT}_{50}$ and $\mathrm{DT}_{90}$ ) for the parent or the TP should be derived (FOCUS 2006).

First, the reports were evaluated whether the labelling position of the radiolabel was given in a comprehensible way. Incomplete study reports and cited literature were discarded. The remaining 33 studies were searched for information on screening for TP, detection, quantification, identification, and $\mathrm{DT}_{x}$ values of TP. The total number of detected TP was recorded, as well as the number of TP present more than once at or above $10 \%$ of the originally applied dose (of radioactivity) for the parent compound. Here also, TP were included that were present at increasing concentrations towards the end of the study. Additionally, the $\mathrm{DT}_{50}$ of the parent and the TP were compared, to identify TP that are longer lived than the respective parent compound. Comparisons were made compartment specific, where possible. In case only a $\mathrm{DT}_{50}$ for the total system was available, the comparison was based on that value. For the comparison in between OECD 308 and 301 results, mineralisation $\left(\mathrm{CO}_{2}\right.$ evolution) on day 28 (or the closest measured day ( \pm 2 days)) was recorded from OECD 308 studies and from the corresponding studies according to OECD 301 on ready biodegradability.

\section{Results}

\section{Ready biodegradability}

Tests according to the OECD guideline $301 \mathrm{~B}$ are suitable to assess the potential of pharmaceutical AI to readily biodegrade. Commonly, only low amounts of mineralisation to $\mathrm{CO}_{2}$ are observed, and AI have to be classified as not readily biodegradable. This was true for all considered small molecule AI. In this article, the term AI refers subsequently to small molecule AI; biologicals were not evaluated. For 29 different $\mathrm{AI}$, the experiments most often report $0 \%$ mineralisation (negative results are set to $0 \%$ ). Therefore, the median of the 29 studies is $0 \%$. The mean is $4 \%$ and the maximal mineralisation observed is $20 \% \mathrm{CO}_{2}$ evolution.

The low mineralisation commonly observed is in accordance with previous findings (Trautwein and Kümmerer 2011; Al-Ahmad et al. 1999). This is not surprising, as pharmaceutical AI are designed to be metabolically stable and thus persistent substances (Nassar et al. 2004). However, the test on ready biodegradability is very useful in excluding AI which are biodegradable and can thus relatively easily be classified as not persistent (like, e.g. many biological AI). However, to assess the persistence of pharmaceutical AI in the environment, simulation studies on transformation of the test substance in environmental compartments like soil, water or water/sediment systems are crucial.

\section{Occurrence of TP}

In all studies in water/sediment systems except for those which did not explicitly contain any information on TP $(n=$ 2 ), TP were detected. The median of the number of detected TP for all studies is 3 , ranging from 1 to 45 . It is important to note that the actual number of formed TP is very likely greater than the number deducible from the reports as (1) TP often cannot be detected due to an unsuitable or not representative labelling position as $\mathrm{AI}$ are often complex molecules (see also comparison 301/308 results below), (2) TP below $10 \%$ are often only summarily treated as "some" or "a few" and have not been counted and (3) only the number of detected peaks is reported. One peak can consist of more than one substance, especially if only low-resolution analytical techniques like thin-layer chromatography are used.

\section{Identification of TP}

In $70 \%$ of studies $(n=22)$, at least one TP $\geq 10 \%$ was detected. It was attempted in $52 \%$ of the studies $(n=16)$ to identify at least one of the TP $\geq 10 \%$. However, in only eight studies $(26 \%)$ were all TP $\geq 10 \%$ identified. Detailed information on detection and attempts on identification of TP in studies conducted according to OECD guideline 308 in water/sediment systems can be found in Fig. 1. Only in about a quarter of all studies are the requirements according to OECD guideline 308 fully met. In a majority of studies, no or only partial information on the identity of TP formed in amounts exceeding $10 \%$ of the originally applied dose is available.

For identification purposes, mostly LC-MS/MS was used. In some cases, reference compounds were available for comparison of retention times. In a few cases, at least for a part of the observed TP, information from the studies on metabolism and excretion from preclinical or clinical studies could be useful for identification of TP. In one case, a TP observed in a study on phototransformation proved helpful 
Fig. 1 Detection and identification in studies conducted according to OECD guideline 308 (water/sediment systems)
$26 \%(n=8)$

at least $1 \mathrm{TP} \geq 10 \%$ detected, all TP $\geq 10 \%$ identified

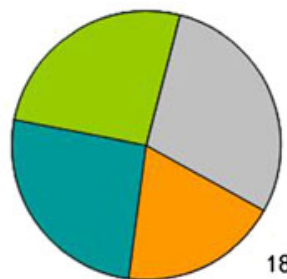

$26 \%(n=8)$ at least 1 TP $\geq 10 \%$ detected, at least 1 TP identified
$18 \%(n=6)$ at least 1 TP $\geq 10 \%$ detected but without identification for identification of a TP from a water/sediment system. However, often, completely different transformation pathways are observed in the environment as compared to human or animal metabolism studies. An example is the different transformation of the AI valsartan. In humans, hydroxylation of the AI is observed (Steinhilber et al. 2010), whereas in the environment, N-dealkylation and amide hydrolysis lead to a different TP (Helbling et al. 2010; Kern et al. 2010). Considering all available information, the following reaction types were observed to occur in the above-mentioned water/sediment systems: oxidation (formation of $\mathrm{C}-\mathrm{C}$ double bonds), hydrolysis (amide hydrolysis, ester hydrolysis), dealkylation ( $\mathrm{N}-, \mathrm{S}-$ ) and reduction ( $\mathrm{S}$ atom). In none of the studies has O-dealkylation been observed, as has already been described by Wick et al. (2011). Wick et al. (2011) only noticed little overlap between transformation pathways in humans and in activated sludge. Löffler et al. (2005) examined a range of AI in OECD 308 studies and did not find similarities between human ME and environmental TP from water/sediment systems. Kern et al. (2010) examined four different AI in batch experiments with activated sludge. Four of the observed TP were identical to previously known human ME, and three TP did not show similarities with human metabolites. Therefore, information from metabolism studies in humans or animals can be helpful when trying to identify environmental TP, but they cannot be used to predict the outcome of studies with environmentally relevant matrices.

\section{Persistence of TP compared to the parent}

For TP that are detected in amounts $\geq 10 \%$ of the originally applied amount of parent substance, according to OECD guideline 308, not only is identification required but also their pattern of formation and decline should be established, which means that a kinetic model has to be fitted to the data to derive a $\mathrm{DT}_{50}$ not only for the parent but also for the TP. This was only accomplished in five of the studies. However, it has to be considered that for eight studies, the amount of TP was increasing towards the study end, and therefore, deriving a $\mathrm{DT}_{50}$ was not possible. In these cases, prolonging the study could have yielded valuable information on the persistence of the TP.

As Fig. 2 illustrates, in $19 \%$ of the studies were TP observed showing a longer $\mathrm{DT}_{50}$ than the parent. For $26 \%$ of the AI, an evaluation of the $\mathrm{DT}_{50}$ is impossible because towards the end of the studies, the concentration of TP is still increasing or at least not showing a clearly declining trend. Consequently, a $\mathrm{DT}_{50}$ cannot be derived. In summary, for $45 \%$ of all $\mathrm{AI}$, there are indications for an increase in $\mathrm{DT}_{50}$ for the TP compared to the parent.

The evaluation highlights that the kinetic fitting of the data is a huge problem regarding the interpretation of the results from simulation studies. Kinetic modelling is most often difficult because too few sampling time points are included in the studies. Recommendations on the evaluation of simulation studies by the FOCUS group (2006) includes that "... the number of sampling times and/or the experimental period may need to be extended, to establish the degradation pathway and distribution between the water column and sediment. ... With metabolites [TP], additional sampling points may be needed to estimate formation rates more precisely, to reduce the influence of the statistical correlation between estimated formation and degradation rates..." Examples for kinetic fitting available on the FOCUS website (2013) for TP include ten sampling time points. As the evaluation shows, for persistent TP, kinetic modelling is often also not possible because the study ends when there are no indications for a decline in concentration of the TP. In these cases, the study should be prolonged or a separate study for the TP should be envisaged. Further difficulties stem from the OECD 308 test system setup. As no clear distinction between partitioning and transformation processes is feasible with the standard setup, fitting of a kinetic model is complicated by the presence of the two compartments water and sediment. Therefore, to gain information on transformation of pharmaceutical AI in surface waters, studies according to OECD 309 (surface water with environmentally relevant particle concentrations as test system without a sediment compartment, OECD 2004) could be an alternative worth exploring.

\section{Comparison of OECD 301 and 308 type studies}

Figure 3 shows data pairs for mineralisation observed in tests according to OECD 301 on ready biodegradability (mostly from OECD $301 \mathrm{~B}$ with $\mathrm{CO}_{2}$ evolution as endpoint) and according to OECD 308 (transformation in water/sediment systems, $\mathrm{CO}_{2}$ evolution, other volatile gases were not observed). 
Fig. 2 Information on $\mathrm{DT}_{50}$ given for TP

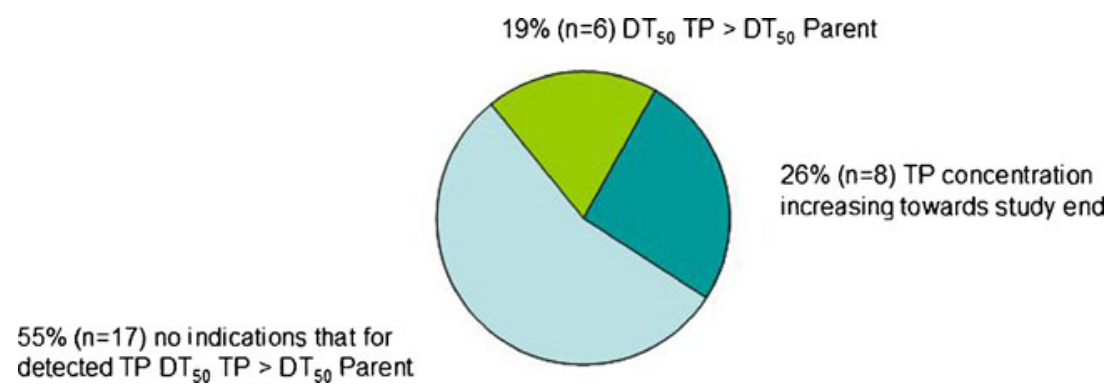

Mineralisation within 28 days from ready biodegradability studies and simulation studies is generally $\leq 20 \%$, underlining the fact that small molecule pharmaceutical AI are predominantly designed to be persistent, stable compounds. It is, however, apparent that there is no correlation between the results of the different studies. A linear regression would yield a correlation coefficient of only 0.1 . To interpret this observation, it is important to consider what the data actually reflect. In tests on ready biodegradability, ultimate biodegradation of the parent to $\mathrm{CO}_{2}$ is measured, taking into account the whole molecule and each $\mathrm{C}$ atom, whereas from the OECD 308 studies, only information on the labelled part of the molecule is obtained. The results show that the information derived from OECD 308 studies differ from the mineralisation unbiased by the labelling position as determined from OECD 301 studies. As pharmaceutical AI are often relatively large, complex molecules, labelling of one phenyl ring or just one $\mathrm{C}$ atom is not enough to get results representative for the whole molecule. This comparison can only be done directly for mineralisation, but it indicates that also for other parameters measured in OECD 308 studies - TP and NER - the same bias has to be assumed that is obvious for mineralisation. The data show that under- as well as overestimation of each parameter may occur when relying on mineralisation results (or results for NER or TP) from insufficiently labelled OECD 308 studies. Besides the problems of representatively radio labelling AI, it is difficult, costly and sometimes barely possible to synthesize suitably ${ }^{14} \mathrm{C}$ radio-labelled material for semi-synthetic molecules like some antibiotic compounds. Consequently, using unlabelled techniques, especially LC-MS/MS-based identification of TP (Prasse et al. 2011; Wick et al. 2011; Stravs et al. 2013), might be more appropriate for detection and identification of TP.

Case study: valsartan and its TP (val)sartan acid

Valsartan is an AI from the sartan class of antihypertensive compounds acting on the $\mathrm{AT}_{1}$ subtype of the angiotensin II receptor (Steinhilber et al. 2010). The class of sartans belongs to the highly consumed pharmaceutical AI (more than $150 \mathrm{t} /$ year in Germany) with valsartan being the most consumed compound (86 t/year in Germany, based on data for 2012 from IMS MIDAS ${ }^{\circledR}$ ). Valsartan has been shown to be transformed to a persistent TP in batch tests using activated sludge (Helbling et al. 2010). The transformation pathway has been elucidated and proceeds via N-dealkylation, subsequent amide hydrolysis and hydrolysis/oxidation to (val)sartan acid (Helbling et al. 2010). The parent compound has a short $\mathrm{DT}_{50}$ which would not lead to concerns about persistence, whereas the TP (val)sartan acid shows a $\mathrm{DT}_{50}$ 410 times longer than that of the parent (Helbling et al. 2010) and consequently fulfils the vP criterion (ECHA 2012) with a $\mathrm{DT}_{50}>60$ days for the water compartment. The parent valsartan has to be considered a mobile substance with a $K_{\mathrm{d}}$ in the range 24-39 L/kg (Kern et al. 2010). As TP are often more polar than their parent compounds, it has to be assumed that (val)sartan acid is also a mobile compound. Due to the high consumption of the parent, its unchanged excretion from patients at about $90 \%$ (Steinhilber et al. 2010), its very high persistence and potential mobility, valsartan acid is likely to pose a risk for groundwater and raw drinking water contamination when drinking water is produced by bank filtration. Based on structural similarity, also other compounds from the sartan class of compounds could form the same TP in the environment. However, there is no experimental evidence for a similar behaviour. (Val)sartan acid has been measured in high concentrations in the range of $1 \mu \mathrm{g} / \mathrm{L}$ in WWTP effluent (Kern et al. 2010). In one of three sampled groundwater wells in Switzerland,

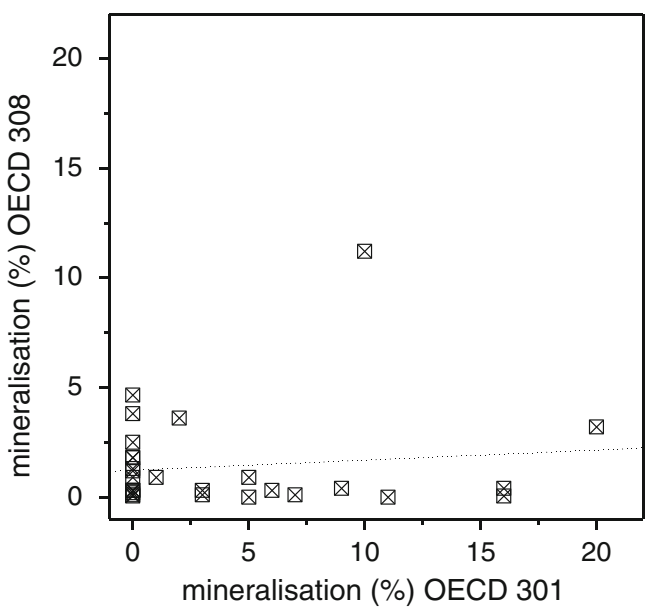

Fig. 3 Comparison of the amount of mineralisation (in percent) from studies according to OECD 301 and OECD 308 after 28 days $(n=29)$. The dashed line represents a linear regression of percent mineralisation in OECD 308 studies vs. OECD 301 studies (coefficient of correlation=0.1) 
concentrations of sartan acid in the low nanogram-per-litre range $(<10 \mathrm{ng} / \mathrm{L})$ were measured. This well is probably influenced by bank filtration from a nearby river (personal communication, Eawag).

The case study of the AI valsartan and its persistent TP (val)sartan acid highlights the fact that, in some cases, it is important to consider TP in the assessment of the behaviour of pharmaceutical $\mathrm{AI}$ in the environment as they might be the more relevant compounds concerning exposure of environmental compartments or the protection of water resources for drinking water production.

\section{Conclusions}

The systematic evaluation of studies on biodegradation and transformation in water/sediment systems shows that pharmaceutical AI are commonly not readily biodegradable and that TP are generally observed. In $45 \%$ of the studies, indications were found that TP show a longer $\mathrm{DT}_{50}$ than the parent compounds. This highlights that only looking at the parent compound is not always sufficient. For these cases (and also cases where parent and TP show a high persistence), TP have to be considered when assessing environmental behaviour and effects of pharmaceuticals regarding (1) ecotoxicological effects, (2) PBT assessment and (3) assessment of persistent and mobile substances with a risk for groundwater or raw drinking water contamination. Especially in the latter two cases, additional information might be required to finalise an environmental risk assessment.

The quality of the studies concerning TP is variable. There is a need for improvement in kinetic fitting especially for TP. Screening for and identification of TP is often problematic and could benefit from rapid development of LCMS/MS techniques and databases for identification purposes based solely on LC-MS spectra and application of these new developments in routine analytical methods. Kinetic modelling could be aided by taking into account the recommendations given by FOCUS (2006), especially including more sampling time points. Test systems like in OECD 309 with low suspended solids concentration might be valuable for assessing the environmental behaviour of pharmaceutical AI in the aquatic compartment; however, there is a lack of experience with this test system. A more detailed understanding of transformation processes for complex molecules like pharmaceuticals at environmentally relevant concentrations and under environmentally relevant conditions could help to improve study design and interpretation in the assessment of transformation and persistence of pharmaceuticals.

The case study on a persistent TP of the antihypertensive AI valsartan clearly illustrates the fact that in some cases, TP have to be considered separately from their parent compounds with regard to their potential for groundwater/raw drinking water contamination. In this respect, it should also be kept in mind that, during drinking water production, some treatment techniques may further transform persistent TP to substances which exhibit (eco)toxicological effects. An example is a toxic ozonation product formed from carboxyaciclovir, a TP of the antiviral AI aciclovir (Prasse et al. 2012) that enters raw drinking water because of its persistence and mobility, or the toxic iodinated disinfection by-products formed in the presence of persistent and mobile iodinated X-ray contrast agents (Duirk et al. 2011).

Acknowledgments We are grateful to my colleagues Arne Hein and Ines Rönnefahrt for the database searches for consumption data and to the anonymous reviewers who helped to improve the manuscript.

Open Access This article is distributed under the terms of the Creative Commons Attribution License which permits any use, distribution, and reproduction in any medium, provided the original author(s) and the source are credited.

\section{References}

Al-Ahmad A, Daschner FD, Kummerer K (1999) Biodegradability of cefotiam, ciprofloxacin, meropenem, penicillin G, and sulfamethoxazole and inhibition of waste water bacteria. Arch Environ Contam Toxicol 37(2):158-163

Bergmann A, Fohrmann R, Weber FA (2011) Zusammenstellung von Monitoringdaten zu Umweltkonzentrationen von Arzneimitteln, Gutachten des IWW Rheinisch-Westfälisches Institut für Wasser Beratungs- und Entwicklungsgesellschaft $\mathrm{mbH}$ im Auftrag des Umweltbundesamtes (in German)

Duirk SE, Lindell C, Cornelison CC, Kormos J, Ternes TA, Attene-Ramos M, Osiol J, Wagner ED, Plewa MJ, Richardson SD (2011) Formation of toxic iodinated disinfection by-products from compounds used in medical imaging. Environ Sci Technol 45(16):6845-6854

EC (2001) Directive 2001/83/EC of the European Parliament and of the Council of 6 November 2001 on the community code relating to medicinal products for human use. Official Journal L-311, 28/11/ 2004, 67-128, as amended

EC (2012) Proposal for a directive amending the WFD and EQSD (COM(2011)876); European Commission: Brussels, 31/01/2012

ECHA (2012) Guidance on information requirements and chemical safety assessment, Chapter R.11: PBT Assessment; ECHA-12-G-24-EN

EMA (2006) Guideline on the environmental risk assessment of medicinal products for human use, EMEA/CHMP/SWP/4447/00

EMA (2010) Questions and answers on 'Guideline on the environmental risk assessment of medicinal products for human use', EMA/ CHMP/SWP/44609/2010

Escher BI, Fenner K (2011) Recent advances in environmental risk assessment of transformation products. Environ Sci Technol 45(9):3835-3847

Escher BI, Bramaz N, Richter M, Lienert J (2006) Comparative ecotoxicological hazard assessment of beta-blockers and their human metabolites using a mode-of-action-based test battery and a QSAR approach. Environ Sci Technol 40(23):7402-7408

Fick J, Soderstrom H, Lindberg RH, Phan C, Tysklind M, Larsson DGJ (2009) Contamination of surface, ground and drinking water from pharmaceutical production. Environ Toxicol Chem 28(12):2522-2527

FOCUS (2006) Guidance document on estimating persistence and degradation kinetics from environmental fate studies on pesticides in EU registration, report of the FOCUS Work Group on Degradation Kinetics, EC Document Reference Sanco/10058/2005 version 2.0 
FOCUS (2013) Website: http://focus.jrc.ec.europa.eu/dk/. Accessed January 2013

Heberer T (2002) Occurrence, fate, and removal of pharmaceutical residues in the aquatic environment: a review of recent research data. Toxicol Lett 131(1-2):5-17

Helbling DE, Hollender J, Kohler HP, Singer H, Fenner K (2010) Highthroughput identification of microbial transformation products of organic micropollutants. Environ Sci Technol 44(17):6621-6627

Hughes SR, Kay P, Brown LE (2013) Global synthesis and critical evaluation of pharmaceutical data sets collected from river systems. Environ Sci Technol 47(2):661-677

Joss A, Zabczynski S, Göbel A, Hoffmann B, Löffler D, McArdell CS, Ternes TA, Thomsen A, Siegrist H (2006) Biological degradation of pharmaceuticals in municipal wastewater treatment: proposing a classification scheme. Water Res 40(8):1686-1696

Kern S, Baumgartner R, Helbling DE, Hollender J, Singer H, Loos MJ, Schwarzenbach RP, Fenner K (2010) A tiered procedure for assessing the formation of biotransformation products of pharmaceuticals and biocides during activated sludge treatment. J Environ Monit 11:2100-2111

Kolpin DW, Furlong ET, Meyer MT, Thurman EM, Zaugg SD, Barber LB, Buxton HT (2002) Pharmaceuticals, hormones, and other organic wastewater contaminants in U.S. streams, 1999-2000: a national reconnaissance. Environ Sci Technol 36(6):1202-1211

Löffler D, Römbke J, Meller M, Ternes TA (2005) Environmental fate of pharmaceuticals in water/sediment systems. Environ Sci Technol 39(14):5209-5218

Nassar AE, Kamel AM, Clarimont C (2004) Improving the decisionmaking process in the structural modification of drug candidates: enhancing metabolic stability. Drug Discov Today 9(23):1020-1028

OECD (1992) OECD guideline for testing of chemicals 301, ready biodegradability. http:/www.oecd-ilibrary.org/environment/oecdguidelines-for-the-testing-of-chemicals-section-3-degradation-andaccumulation 2074577x. Accessed 28 Jan 2013

OECD (2002) OECD Guideline for the testing of chemicals 308, Aerobic and Anaerobic Transformation in Aquatic Sediment Systems. http://www.oecd-ilibrary.org/environment/oecd-guidelines-for- the-testing-of-chemicals-section-3-degradation-and-accumulation 2074577x. Accessed 28 Jan 2013

OECD (2004) OECD guideline for the testing of chemicals 309, aerobic mineralisation in surface water-simulation biodegradation test. http://www.oecd-ilibrary.org/environment/oecd-guidelines-for-thetesting-of-chemicals-section-3-degradation-and-accumulation 2074577x. Accessed 28 Jan 2013

Phillips PJ, Smith SG, Kolpin DW, Zaugg SD, Buxton HT, Furlong ET, Esposito K, Stinson B (2010) Pharmaceutical formulation facilities as sources of opioids and other pharmaceuticals to wastewater treatment plant effluents. Environ Sci Technol 44(13):4910-4916

Prasse C, Wagner M, Schulz R, Ternes TA (2011) Biotransformation of the antiviral drugs acyclovir and penciclovir in activated sludge treatment. Environ Sci Technol 45(7):2761-2769

Prasse C, Wagner M, Schulz R, Ternes TA (2012) Oxidation of the antiviral drug acyclovir and its biodegradation product carboxyacyclovir with ozone: kinetics and identification of oxidation products. Environ Sci Technol 46(4):2169-2178

Sanchez W, Sremski W, Piccini B, Palluel O, Maillot-Maréchal E, Betoulle S, Jaffal A, Aït-Aïssa S, Brion F, Thybaud E, Hinfray N, Porcher JM (2011) Adverse effects in wild fish living downstream from pharmaceutical manufacture discharges. Environ Int 37(8): 1342-1348

Steinhilber D, Schuster-Zsilavecz M, Roth HJ (2010) Medizinische Chemie, 2nd edn. Deutscher Apotheker Verlag, Stuttgart (in German)

Stravs MA, Schymanski EL, Singer HP, Hollender J (2013) Automatic recalibration and processing of tandem mass spectra using formula annotation. J Mass Spectrom 48(1):89-99

Ternes TA (1998) Occurrence of drugs in German sewage treatment plants and rivers. Water Res 32(11):3245-3260

Trautwein C, Kümmerer K (2011) Incomplete aerobic degradation of the antidiabetic drug metformin and identification of the bacterial dead-end transformation product guanylurea. Chemosphere 85(5):765773

Wick A, Wagner M, Ternes TA (2011) Elucidation of the transformation pathway of the opium alkaloid codeine in biological wastewater treatment. Environ Sci Technol 45(8):3374-3385 